\title{
Revisiting moment-based characterization for wind pressures
}

Guoqing Huang ${ }^{1}$, Ying Luo ${ }^{2}$, Kurtis R. Gurley ${ }^{3}$, Jie Ding $^{4}$

${ }^{1,2}$ School of Civil Engineering, Southwest Jiaotong University, Chengdu, China 610031

${ }^{3}$ Department of Civil and Coastal Engineering, University of Florida, Gainesville, FL, USA 32611

${ }^{4}$ Department of Civil, Environmental and Construction Engineering, Texas Tech University, Lubbock, TX, USA 79409

\section{Abstract}

The estimation of peak wind pressures is important in the reliability- and performance-based design for low-rise buildings. Typically, Davenport's formula is widely used to determine the peak factor if the pressure approximately follows Gaussian distribution. Recently, the moment-based Hermite polynomial model (HPM) is becoming popular to estimate the peak factor when the non-Gaussianity of wind pressure exists. However, their performances deserve further study based on the appropriate wind tunnel data. In this study, the Davenport's formula and moment-based HPM are reviewed. The peak value of wind pressure is determined using very long time histories of wind pressure data to evaluate the performance of moment-based HPM and Davenport's formula. Results suggest that moment-based HPM should be adopted in the peak value estimation for wind pressures when the skewness and kurtosis of a process are sufficient to capture its non-Gaussian properties. Results also show that Davenport's formula may cause noticeable errors in 
the peak factor estimation for the wind pressure data close to Gaussian process while HPM provides a robust estimation for these data.

Keywords: Wind pressure; Non-Gaussian process; Hermite polynomial model; Skewness; Kurtosis; Peak factor; Peak value

\section{Introduction}

The low-rise building roof is very vulnerable to strong winds, thus the characterization of wind pressures on the roof top is an important issue in the reliability- and performance-based design for low-rise buildings. In most design codes and specifications, the mean value of the peak wind pressure during the duration $T$ is determined from the peak factor, which is defined as

$$
g=\left(\mu_{x_{p}}-\mu_{x}\right) / \sigma_{x}
$$

where $\mu_{x_{p}}$ represents the mean of peak values for wind pressure coefficient $X(t)$, and $\mu_{x}$ and $\sigma_{x}$ are the mean and standard deviation (STD) of $X(t)$, respectively. Note that the peak value selected from each segment is the instantaneous maximal value from the raw data, and not averaged for a short duration, such as 3 seconds in full scale. When the wind pressure approximately follows the Gaussian distribution, the well-known Davenport's formula can be used to determine the peak factor. However, significant portions of wind pressures on the roof top may exhibit non-Gaussianity and it has been shown that the application of Davenport's formula to non-Gaussian data can lead to unconservative peak factor estimates (e.g., Balderrama 
et al. 2012).

Based on whether kurtosis is larger than 3, the non-Gaussian process is classified as "hardening" (kurtosis< 3) and "softening" (kurtosis> 3) cases (Winterstein 1988). "Hardening" wind pressure processes may have bimodal distributions (Ding and Chen 2014), and skewness and kurtosis may not be effective to characterize these non-Gaussian features. The peak factor of the "Hardening" wind pressure process is usually less than that of a Gaussian process and the application of Davenport's formula is conservative in the cladding design. Therefore the "softening" wind pressure is the focus hereafter. For "softening" processes, the peak factor can be calculated by moment-based Hermite polynomial model (HPM) through closed-form formula. Based on skewness and kurtosis, Kareem and Zhao (1994), Chen and Huang (2009) and Kwon and Kareem (2011) derived the closed-form formula, while Huang et al. (2013) obtained it from the probability function of local peaks of wind pressures, which was fitted by Weibull distribution. If the peak wind pressure follows Gumbel distribution, the peak factor is associated with the peak wind pressure at $57 \%$ fractile. Note that fractile values presented in this study are referring to the distribution of the peak pressure and the peak value is selected from the pressure time history of duration $T$ where $T$ could be 10 -min or 1 hour. Due to the conservativeness and simplicity, Holmes and Cochran (2003) recommended the use of Gumbel distribution. $78 \%$ fractile of the peak pressure coefficient is suggested for defining design wind loading in codes and standards to consider the uncertainty of both wind speed and pressure coefficient (e.g., Cook 1990; Chen and Huang 2010). Focusing on 78\% 
fractile peak pressure coefficient for non-Gaussian roof pressure data, Peng et al. (2014) demonstrated moment-based HPM has the satisfactory performance based on the closed-form approximate solution to HPM coefficients by Yang et al. (2013). Although both of Davenport's formula and moment-based HPM have been studied previously (e.g., Balderrama et al. 2012; Peng et al. 2014; Huang et al. 2015), there is a need to systematically scrutinize their performance in estimating the peak factor and peak value using very long wind pressure data.

In this study, the Davenport's formula and moment-based HPM are reviewed, and some new insights are presented. Using very long wind pressure data collected by the University of Western Ontario (UWO), the peak values of wind pressures are quantified empirically. Then the ability of moment-based HPM and Davenport's formula to estimate the empirical peak factor and peak value is evaluated in detail. Finally, concluding remarks will be given.

\section{Davenport's formula}

For the standard Gaussian process $U(t)$, the cumulative distribution function (CDF) of the peak value during the time period $T$ is given as follows

$$
F_{U_{p}}\left(u_{p}\right)=\exp \left[-v_{0} T \exp \left(-\frac{u_{p}^{2}}{2}\right)\right]
$$

and the corresponding probability density function (PDF) is given by

$$
f_{U_{p}}\left(u_{p}\right)=v_{0} T u_{p} \exp \left[-v_{0} T \exp \left(-\frac{u_{p}^{2}}{2}\right)-\frac{u_{p}^{2}}{2}\right]
$$

where $U_{p}$ is the peak value of the process $U(t)$ during $T$ and $u_{p}$ are specific 
87

88

values of $U_{p}$, and $v_{0}$ is the mean upcrossing rate across zero. The upcrossing rate $v_{0}$ can be computed by

$$
v_{0}=\sqrt{\int_{0}^{\infty} f^{2} S_{U}(f) d f} / \sqrt{\int_{0}^{\infty} S_{U}(f) d f}
$$

where $f$ is the frequency and $S_{U}(f)$ is the power spectral density (PSD) of $U(t)$.

$$
\text { If } \xi=v_{0} T \exp \left(-\frac{u_{p}^{2}}{2}\right) \text { is introduced, then } u_{p}=\sqrt{2 \ln \left(v_{0} T\right)-2 \ln \xi} \text {. Based on }
$$

Taylor's theorem, $u_{p}$ is expressed as

$$
u_{p}=\beta-\frac{\ln \xi}{\beta}-\frac{\ln ^{2} \xi}{2 \beta^{3}}+\cdots
$$

where $\beta=\sqrt{2 \ln \left(v_{0} T\right)}$. The mean and standard deviation (STD) of the peak value can be derived as

$$
\begin{gathered}
\mu_{u_{p}}=\int_{0}^{\infty} u_{p} d F_{U_{p}}\left(u_{p}\right)=\int_{0}^{\infty} u_{p} \exp (-\xi) d \xi \\
\sigma_{u_{p}}=\sqrt{\int_{0}^{\infty}\left(u_{p}-\mu_{u_{p}}\right)^{2} d F_{U_{p}}\left(u_{p}\right)}=\sqrt{\int_{0}^{\infty}\left(u_{p}-\mu_{u_{p}}\right)^{2} \exp (-\xi) d \xi}
\end{gathered}
$$

Neglecting the terms of order $\beta^{-3}$ and substituting Eq. (5) to Eqs. (6) and (7) respectively, the mean and STD of the peak value can be determined by (Davenport 1964)

$$
\mu_{u_{p}}=\beta+\frac{\gamma}{\beta}
$$

$$
\sigma_{u_{p}}=\frac{\pi}{\sqrt{6} \beta}
$$

103 Where $\gamma \approx 0.5772$ is Euler's constant. It is well known that $g=\mu_{u_{p}}$ is the 
derived from the perspective of Gumbel distribution because the peak value

107 distribution of $U(t)$ approximately follows Gumbel distribution. The CDF of the peak value during the time period $T$ can be approximately expressed as

$$
F_{U_{p}}\left(u_{p}\right)=\exp \left\{-\exp \left[-\alpha_{u_{p}}\left(u_{p}-\widehat{u}_{p}\right)\right]\right\}
$$

and the corresponding PDF is given by

$$
f_{U_{p}}\left(u_{p}\right)=\alpha_{u_{p}} \exp \left\{-\exp \left[-\alpha_{u_{p}}\left(u_{p}-\widehat{u}_{p}\right)\right]-\alpha_{u_{p}}\left(u_{p}-\widehat{u}_{p}\right)\right\}
$$

where the dispersion $1 / \alpha_{u_{p}}$ is a measure of "spread"; and the mode $\widehat{u}_{p}$ is the value with maximum likelihood.

Based on the assumption that the CDF defined by Eq. (2) and its derivative are equal to those associated with Eq. (10) at the mode $u_{p}=\widehat{u}_{p}$, the following simultaneous equations can be obtained

$$
v_{0} T \exp \left(-\frac{\widehat{u}_{p}^{2}}{2}\right)=1 ; \quad v_{0} T \hat{u}_{p} \exp \left(-\frac{\widehat{u}_{p}^{2}}{2}\right)=\alpha_{u_{p}}
$$

Hence

$$
\widehat{u}_{p}=\sqrt{2 \ln \left(v_{0} T\right)}=\beta ; \quad \alpha_{u_{p}}=\beta
$$

and the mean and STD of the peak value are given by

$$
\mu_{u_{p}}=\widehat{u}_{p}+\frac{\gamma}{\alpha_{u_{p}}}=\beta+\frac{\gamma}{\beta}
$$

$$
\sigma_{u_{p}}=\frac{\pi}{\sqrt{6} \alpha_{u_{p}}}=\frac{\pi}{\sqrt{6} \beta}
$$


can be determined as

$$
u_{p, q}=\sqrt{2 \ln \frac{v_{0} T}{\ln (1 / q)}}
$$

Because the peak value of $U(t)$ approximately follows Gumbel distribution, the peak factor can be readily estimated by substituting $q=57 \%$ to Eq. (16). Also other $q$ fractile values can be obtained, such as $78 \%$ and $86 \%$ fractiles.

\section{Moment-based HPM}

As mentioned previously, $X(t)$ represents wind pressure coefficient process. Accordingly, the standardized process is expressed by $\tilde{X}(t)=\left[X(t)-\mu_{x}\right] / \sigma_{x}$. For the "softening" case, the following third order HPM is used to relate the standard Gaussian process $U(t)$ and the standardized non-Gaussian process $\tilde{X}(t)$ (Winterstein 1988):

$$
\tilde{x}=H(u)=\kappa\left[H_{1}(u)+h_{3} H_{2}(u)+h_{4} H_{3}(u)\right]
$$

where $\kappa=1 / \sqrt{1+2 h_{3}^{2}+6 h_{4}^{2}}$ is a scaling factor to ensure that $\tilde{X}(t)$ has unit variance; $h_{3}$ and $h_{4}$ are parameters which control the shape of the distribution of $\tilde{X}(t)$; the $i$ th Hermite polynomial function is defined as

$$
H_{i}(u)=(-1)^{i} \exp \left(\frac{u^{2}}{2}\right) \frac{d^{i}}{d u^{i}}\left[\exp \left(-\frac{u^{2}}{2}\right)\right]
$$

More specifically, $H_{1}(u)=u, H_{2}(u)=u^{2}-1$ and $H_{3}(u)=u^{3}-3 u$.

The parameters can be obtained through numerical solution of the nonlinear equations derived by Tognarelli et al. (1997) and Gurley (1997), or by Ditlevsen et al. (1996). Both sets of equations can be found in Appendix A, where the equivalence between them is also illustrated. To improve the computational efficiency, several 
closed-form approximate formulas have been proposed to estimate the parameters, including Winterstein (1988), Winterstein and Kashef (2000) and Yang et al. (2013). The approximate solution by Yang et al. (2013) has been shown to perform well for mildly and strongly non-Gaussian wind pressure. In this study, the nonlinear equations will be solved via Newton-Raphson iteration to obtain the parameters directly.

According to Eq. (17), $U(t)$ can be expressed by the function of $\tilde{X}(t)$ as (Winterstein 1988)

$$
u=H^{-1}(\tilde{x})=\left[\sqrt{\zeta^{2}(\tilde{x})+c}+\zeta(\tilde{x})\right]^{-/ 3}-\left[\sqrt{\zeta^{2}(\tilde{x})+c}-\zeta(\tilde{x})\right]^{-/ 3}-a
$$

where $\zeta(\tilde{x})=1.5 b\left(a+\frac{\tilde{x}}{\kappa}\right)-a^{3}, a=\frac{h_{3}}{3 h_{4}}, b=\frac{1}{3 h_{4}}$, and $c=\left(b-1-a^{2}\right)^{3}$.

Clearly, the inverse function $H^{-1}(\cdot)$ requires the monotonicity of Eq. (17). Choi and Sweetman (2010) gave the following condition which should be satisfied

$$
h_{3}^{2} \leq 3 h_{4}\left(1-3 h_{4}\right)
$$

Winterstein and MacKenzie (2013) proposed the approximate inequality which is related with skewness and kurtosis

$$
3+\left(1.25 \alpha_{3}\right)^{2} \leq \alpha_{4}
$$

where $\alpha_{3}$ and $\alpha_{4}$ are skewness and kurtosis of $X(t)$, respectively.

Substituting Eq. (19) into Eq. (2), the peak value distribution of $\tilde{X}(t)$ during the time period $T$ can be calculated as follows

$$
F_{\bar{X}_{p}}\left(\tilde{x}_{p}\right)=F_{U_{p}}\left(u_{p}\right)=\exp \left\{-v_{0} T \exp \left\{-\frac{\left[H^{-1}\left(\tilde{x}_{p}\right)\right]^{2}}{2}\right\}\right\}
$$

165 where $\tilde{X}_{p}$ is the peak value of the process $\tilde{X}(t)$ during $T$ and $\tilde{x}_{p}$ is the dummy 
166

167

168

169

170

171

172

173

174

175

176

177

178

179

$$
\mu_{\tilde{x}_{p}}=\kappa\left\{\left(\beta+\frac{\gamma}{\beta}\right)+h_{3}\left(\beta^{2}+2 \gamma-1+\frac{1.98}{\beta^{2}}\right)\right.
$$

$$
\left.+h_{4}\left[\beta^{3}+3 \beta(\gamma-1)+\frac{3}{\beta}\left(\frac{\pi^{2}}{6}-\gamma+\gamma^{2}\right)+\frac{5.44}{\beta^{3}}\right]\right\}
$$

180 In addition, they proposed the following equation for the STD of the peak value of $181 \tilde{X}(t)$ by substituting Eqs. (5) and (17) into Eq. (24)

182

$$
\sigma_{\tilde{x}_{p}}=\kappa\left[\frac{\pi^{2}}{6 \beta^{2}}+6.58 h_{3}^{2}+9 h_{4}^{2}\left(1.64 \beta^{2}+\frac{12.69}{\beta^{2}}+5.32\right)\right.
$$

$$
\left.+\frac{6.58}{\beta} h_{3}+6 h_{4}\left(\frac{2.66}{\left(\beta^{2}\right.}+1.64\right)+12 h_{3} h_{4}\left(1.64 \beta+\frac{2.66}{\beta}\right)\right]^{1 / 2}
$$


Meanwhile, Chen and Huang (2009) estimated the mean of the peak value of $\tilde{X}(t)$ by directly substituting that of $U(t)$ into Eq. (17) and suggested the following slightly different closed-form formula

$$
\mu_{\tilde{x}_{p}}=H\left(\mu_{u_{p}}\right) \approx \kappa\left\{\left(\beta+\frac{\gamma}{\beta}\right)+h_{3}\left(\beta^{2}+2 \gamma-1\right)+h_{4}\left[\beta^{3}+3 \beta(\gamma-1)\right]\right\}
$$

Some minor terms are neglected because their contribution to the mean is small.

Since $\tilde{X}(t)$ is the standardized non-Gaussian process, $g_{N}=\mu_{\tilde{x}_{p}}$ is the peak factor of $X(t)$. When $X(t)$ follows the Gaussian distribution, Eqs. (25), (26) and (28) become $\beta+\gamma / \beta$ as $h_{3}=h_{4}=0$ and $\kappa=1$. Similarly, Eq. (27) reduces to $\pi /(\sqrt{6} \beta)$ for Gaussian processes. The equations mentioned above are all proposed to obtain the positive peak factor. Thus when the negative wind pressure is analyzed, the pressure time history should be multiplied by -1 .

More generally, the $q$ fractile of the peak value of $\tilde{X}(t)$ can be computed by substituting Eq. (16) into Eq. (17)

$$
\tilde{x}_{p, q}=H\left(u_{p, q}\right)=\kappa\left[u_{p, q}+h_{3}\left(u_{p, q}^{2}-1\right)+h_{4}\left(u_{p, q}^{3}-3 u_{p, q}\right)\right]
$$

Similar to Gaussian process, the peak factor can be approximately evaluated by applying the $57 \%$ fractile of the peak value of $U(t)$ to Eq. (29). Accordingly, the $q$ fractile peak value of $X(t)$ can be expressed by the following equation

$$
x_{p, q}=\mu_{x}+\tilde{x}_{p, q} \sigma_{x}
$$

\section{Wind pressure data}

The wind tunnel test was conducted in Boundary Layer Wind Tunnel II at the University of Western Ontario (UWO), consisting of two 1:50 scale house models (FL27 and FL30) in suburban exposure. Each model includes the terrain without 
neighboring houses and the terrain with neighboring houses. For FL27 house model, there are 496 taps on the roof including three bad taps. FL30 house model contains 474 taps on the roof without any bad taps. The two house models are illustrated in Figure 1. The layout of the corresponding taps can be seen in Figure 2, where the selected taps in the following discussion are also plotted. The details can be found in Peng et al. (2014). The sampling frequency for model scale is $400 \mathrm{~Hz}$. For wind directions of $120^{\circ}, 125^{\circ}$, and $130^{\circ}$, the sampling time for model scale is $3 \mathrm{~h}$. Based on the assumption that a full-scale wind speed at $10 \mathrm{~m}$ height in suburban exposure is $31.7 \mathrm{~m} / \mathrm{s}$, the velocity scale is determined as 1:5. Accordingly, the sampling frequency and time of these wind directions for full scale are $40 \mathrm{~Hz}$ and $30 \mathrm{~h}$, respectively. The very long data $(30 \mathrm{~h})$ are divided into 180 segments of $10 \mathrm{~min}$ duration in the following analysis. In total, 5802 tap records are available, including the two models, two terrains and three wind directions. The taps selected for analysis in this study are summarized in Table 1, including the model, terrain and wind direction. In Table 1, "I" and " $\mathrm{H}$ " represent the terrain without neighboring houses and the counterpart with neighboring houses, respectively.

\section{Peak wind pressure based on wind tunnel data}

In this study, the number "-1" has been multiplied to time histories of wind pressure coefficients. The means of skewness and kurtosis from 180 segments of wind pressure coefficients associated with all the taps are depicted in Figure 3. It can be seen that the kurtosis value is almost always larger than 3 . Also the effective regions expressed by Eqs. (20) and (21) are plotted in Figure 3. Only 9.9\% of all the taps are outside of the effective region, and these taps are very close to the border. For these taps, the vertical mapping recommended by Peng et al. (2014) is adopted in this study. In this mapping, the kurtosis is maintained and the skewness is recalculated by 

"softening" processes to evaluate the peak value distribution.

In following discussions about the peak value of the wind pressure coefficient,

233 the standardized time history $\tilde{X}(t)$ is used so that the influence of the mean and STD of wind pressures on the peak values can be eliminated. The relationship between the original time history $X(t)$ and $\tilde{X}(t)$ can be found in Appendix B.

For each tap, the largest value can be collected from each of the 180 segments of standardized wind pressure coefficient data. By averaging the largest values, the peak factor for each tap can be determined, which is shown in Figure 4(a). Dotted lines are used to define the contour range. Also an empirical CDF can be established based on these largest values. Then any fractile of the peak value can be evaluated from this CDF via linear interpolation. For instance, $57 \%, 78 \%$ and $86 \%$ fractiles of the peak values of standardized wind pressure coefficients for all taps using wind tunnel data are shown in Figure 4(b)-(d), respectively. It is obvious that Figure 4(a) and Figure 4(b) are very similar. The difference between the peak factor and peak value at $57 \%$ fractile is within $5 \%$ for $99.7 \%$ of the taps. This means that Gumbel distribution is an appropriate candidate to fit the peak wind pressure coefficient. Given the skewness and kurtosis, the peak factor and peak values at $57 \%, 78 \%$ and $86 \%$ fractiles for a segment of standardized wind pressure time history can be approximately determined from Figure 4. Note that the sampling frequency or duration will also influence peak values of the process, which has been discussed in Sadek and Simiu (2002). If the peak value distribution follows Gumbel distribution, the peak value at any fractile can be interpolated from the plots in Figure 4.

Results show that the increase of skewness (with kurtosis constant) does not always lead to a larger peak factor, while the theoretical solution from moment-based 
HPM shows the peak factor will increase with the increase of skewness. Taps 1-3 are selected for illustration. Their peak factors along with the skewness and kurtosis are summarized in Table 2. The taps with larger skewness have lower peak factors. This can be observed in their standardized time histories as shown in Figure 5. It can be seen that the time history with similar kurtosis but larger skewness has more frequency but smaller outlier values within a given duration. Hence, the peak factor may be smaller, for larger skewness with the same kurtosis. This represents an exception rather than an expected behavior, nonetheless, it illustrates a limitation of relying on higher moments exclusively to quantify non-Gaussian behavior. Note that these peak factors are significantly larger than that from the Davenport's formula, which is mainly attributed to the large kurtosis.

\section{Performance of moment-based HPM}

Each of the closed-form formulas [Eqs. (25), (26) and (28)] can be applied to 180 10-min duration segments of standardized wind pressure coefficients to estimate 180 peak factors. To estimate the peak value at any fractile, moment-based HPM can be applied to these segments to estimate 180 peak CDFs, from which the peak values at 57\% and other fractiles can be determined (e.g., Peng et al. 2014). In this study, Eq. (29) can be used conveniently to estimate peak values at these fractiles. Accordingly, the mean, among the 180 segments, of peak factors and peak values can be obtained. Results show that the differences for the peak factors by different closed-form formulas are within $1.5 \%$. Further comparison shows that the difference between the peak factor by Eq. (26) and peak value at $57 \%$ fractile by Eq. (29) is within $4 \%$. Hence, closed-form formulas and Eq. (29) at 57\% fractile provide almost identical 
solutions for the peak factor.

In following discussion, the evaluation of HPM performance will be based on the relative error, which is defined as

$$
e_{H}=\tilde{x}_{p}^{H} / \tilde{x}_{p}^{w}-1
$$

where $\tilde{x}_{p}^{H}$ denotes the mean peak factor by Eq. (26) or mean peak value at any fractile by Eq. (29), and $\tilde{x}_{p}^{w}$ is the corresponding peak factor or peak value from the wind tunnel data. The errors associated with HPM are shown in Figure 6.

Table 3 provides the breakdown of error ranges, as averaged over all 180 10-min segments for each tap. The number of taps with higher errors grows slightly as the fractile value increases. The estimates for majority of the taps fall within $+/-10 \%$ of the empirical value, validating the efficacy of the HPM based estimation method. However, estimates at a minority of taps produce larger errors. These cases are examined in more detail to illustrate the potential limitations of the method.

Tap 4 is selected for examination, where HPM underestimates the peak factor by an average of $18.5 \%$ among all 180 10-min segments. The PDFs of two typical 10-min segments for tap 4 are displayed in Figure 7. It can be seen that HPM has a lighter weight in the tail than the data. This is typical of the majority of the 180 10-min segments at tap 4, thus producing the underestimation for peak values. Figure 8 shows the PDFs of two 10-min segments associated with tap 5, where HPM overestimates the peak factor by an average of $25.7 \%$ among all 18010 -min segments, producing a mean overestimation.

For peak factor and various fractile values, the percentages of taps associated 
with different relative error ranges are summarized in Table 3. It is seen that HPM provides a good estimation for the majority of wind pressures. Hence the moment-based HPM can be considered to be a good tool to estimate the peak values. However, the above discussion highlights that the method is not guaranteed to be robust in all scenarios. As a final point of consideration, the HPM as applied in this study uses the method of moments to determine the Hermite parameters as per section 3. The HPM may also be fitted to the data via maximum likelihood, which would remove the heavy reliance on the higher moments to describe the probability content of the data. Although not investigated herein, this has the potential to improve the performance of the HPM peak estimation model.

\section{Validity of Davenport's formula}

To simplify cladding design, the wind pressure on the roof is commonly classified into Gaussian and non-Gaussian zones based on the skewness and kurtosis. When the time history of the wind pressure coefficient is located within the Gaussian zone, the peak factor is determined by Davenport's formula. Otherwise, other approaches such as moment-based HPM should be used. In this section, the validity of this partitioning approach is examined.

A skewness and kurtosis region of $0-1$ and 3-4.5, respectively, is selected for the sake of discussion, as shown in Figure 9. This region might reasonably be considered mildly non-Gaussian in the extreme (upper right), and essentially Gaussian in the lower left. Figure 9 also shows the mean skewness and kurtosis of wind pressure coefficients from the dataset (averaged from 180 segments), located in the aforementioned region. The sufficient number of taps with data that falls within the 
rectangular region provides a good chance to evaluate the adequacy of Davenport's formula for peak factor estimation of mildly non-Gaussian data.

Similar to the evaluation of HPM, the validity of Davenport's formula is also examined by the relative error, which is defined as

$$
e_{D}=g^{D} / g^{w}-1
$$

where $g^{D}$ and $g^{w}$ denote the mean peak factor calculated by Davenport's formula and corresponding empirical peak factor from wind tunnel data, respectively. The errors resulting from Davenport's formula are depicted in Figure 10(a). It is found that Davenport's formula may underestimate the peak factor by more than $40 \%$ and up to $15 \%$ in the lower left region close to Gaussian process. Similar comparison of Davenport's formula with other peak factor estimation methods was thoroughly covered in Balderrama et al. (2012) for hurricane wind speeds. It was found that Davenport's formula leads to unconservative peak factor estimation for non-Gaussian data. The errors resulting from HPM estimation applied to the same data are plotted in Figure 10(b). Results show that HPM performs well relative to Davenport's formula, with errors less than $10 \%$ relative to the empirical for almost all taps in the region. A comparison between Figures $10 \mathrm{a}$ and $10 \mathrm{~b}$ is presented in Figure 11 as the ratios of peak factors from Davenport's formula to HPM counterparts via Eq. (26) within the effective (monotonic) region of HPM. Davenport's formula quickly degrades in accuracy as departures from the Gaussian increase. This illustrates that the division into Gaussian and non-Gaussian zones has little utility for peak factor estimation. HPM is well suited as a robust estimator of peak factors for Gaussian, mildly non-Gaussian, strongly non-Gaussian data that fall within the effective region 


\section{Conclusions}

In this study, the Davenport's formula and moment-based HPM were revisited, and some new insights were addressed. Davenport's formula was derived from the perspective of Gumbel distribution. The equivalence between two sets of well-known nonlinear equations for determining HPM parameters was discussed.

Based on standardized very long duration wind pressure data, the empirical and estimated peak factor and peak values at $78 \%$ and $86 \%$ fractiles were presented. The accuracy of HPM was evaluated. Results showed moment-based HPM performed well as a whole. However, HPM could not perform well for the wind pressures outside of its effective region. HPM underperformed in rare cases even within the effective region. peak factor in region considered to be mildly non-Gaussian and up to $15 \%$ in the region close to Gaussian process. When HPM was applied to the same data, results showed HPM had the robust performance.

\section{Acknowledgments} Natural Science Foundation of China (Grant No. 51478401) are greatly acknowledged. The authors are very grateful for the wind pressure data produced by the University of 


\section{References}

369

370

371

372

373

374

375

376

377

378

379

380

381

382

383

384

385

388

Balderrama, J.A., Masters, F.J., and Gurley, K.R. (2012). "Peak factor estimation in hurricane surface winds.” J. Wind Eng. Ind. Aerodyn., 102, 1-13.

Chen, X., and Huang, G. (2009). "Evaluation of peak resultant response for wind-excited tall buildings.” Eng. Struct., 31(4), 858-868.

Chen, X., and Huang, G. (2010). "Estimation of Probabilistic Extreme Wind Load Effects: Combination of Aerodynamic and Wind Climate Data." J. Eng. Mech., 136(6), 747-760.

Choi, M., and Sweetman, B. (2010). "The Hermite moment model for highly skewed response with application to tension leg platforms.” J. Offshore Mech. Arctic Eng., 132(2), 021602.

Cook, N.J., and Mayne, J.R. (1979). “A novel working approach to the assessment of wind loads for equivalent static design.” J. Ind. Aerodyn., 4, 149-164.

Cook, N. J. (1990). The designer's guide to wind loading of building structures, Part 2: static structures. Building Research Establishment Report, Garston, UK.

Davenport, A. G. (1964). "Note on the distribution of the largest value of a random function with application to gust loading." Proc., Instit. Civil Eng., 28, 187-196.

Ding, J., and Chen, X. (2014). “Assessment of methods for extreme value analysis of non-Gaussian wind effects with short-term time history samples.” Eng. Struct., 80, $75-88$.

Ditlevsen, O., Mohr, G., and Hoffmeyer, P. (1996). "Integration of non-Gaussian fields.” Prob. Eng. Mech., 11, 15-23. 
390

391

392

393

394

395

396

397

398

399

400

401

402

403

404

405

406

407

408

409

410

411

Grigoriu, M. (1984). "Crossings of non-Gaussian translation processes.” J. Eng.

Mech., 110(4), 610-620.

Gurley, K.R. (1997). "Modelling and simulation of non-Gaussian processes.” Ph.D.

Dissertation, University of Notre Dame.

Holmes, J.D., and Cochran, L.S. (2003). "Probability distributions of extreme pressure coefficients.” J. Wind Eng. Ind. Aerodyn., 91(7), 893-901.d

Huang G., He H., Mehta K.C. and Liu X. (2015). “Data-based probabilistic damage estimation for asphalt shingle roofing.” J. Struct. Eng., 141(12), 04015065.

Huang, M., Lou, W., Chan, C.M., Lin, N., and Pan, X. (2013). "Peak distributions and peak factors of wind-induced pressure processes on tall buildings.” J. Eng. Mech., 139(12), 1744-1756.

Kareem, A., and Zhao, J. (1994). "Analysis of Non-Gaussian Surge Response of Tension Leg Platforms Under Wind Loads.” J. Offshore Mech. Arctic Eng., 116, 137-144.

Kwon, D.K., and Kareem, A. (2011). "Peak factors for non-Gaussian load effects revisited.” J. Struct. Eng., 137(12), 1611-1619.

Peng, X., Yang, L., Gavanski, E., Gurley, K., and Prevatt, D. (2014). “A comparison of methods to estimate peak wind loads on buildings." J. Wind Eng. Ind. Aerodyn., $126,11-23$

Sadek, F., and Simiu, E. (2002). "Peak non-Gaussian wind effects for database-assisted low-rise building design.” J. Eng. Mech., 2002, 128(5), 530-539.

Tognarelli, M.A., Zhao, J., and Kareem, A. (1997). "Equivalent statistical 
412

413

414

415

416

417

418

419

420

421

422

423

424

425

426

427

428

429

430

431

432

cubicization for system and forcing nonlinearities.” J. Eng. Mech., 123(8), 890-893.

Winterstein, S.R. (1988). "Nonlinear vibration models for extremes and fatigue." J. Eng. Mech., 114(10), 1772-1790.

Winterstein, S.R., and Kashef, T. (2000). "Moment-based load and response models with wind engineering applications.” J. Solar Energy Eng., 122(3), 122-128.

Winterstein, S.R., and MacKenzie, C.A. (2013). "Extremes of nonlinear vibration: comparing models based on moments, 1-moments, and maximum entropy." J. Offshore Mech. Arctic Eng., 135(2), 021602.

Yang, L., Gurley, K.R., and Prevatt, D.O. (2013). "Probabilistic modeling of wind pressure on low-rise buildings.” J. Wind Eng. Ind. Aerodyn., 114, 18-26.

Yang, Q., and Tian, Y. (2015). "Model of probability density function of non-Gaussian wind pressure with multiple samples.” J. Wind Eng. Ind. Aerodyn., 140, $67-78$.

\section{Appendix A}

According to a Taylor series, the $i$ th Hermite polynomial of the standardized non-Gaussian process $\tilde{X}(t), H_{i}(\tilde{x})$, can be approximately evaluated at $\tilde{x}_{0}=\kappa u$ and expressed as

$$
H_{i}(\tilde{x}) \approx H_{i}(\kappa u)+\sum_{j=1}^{n} \frac{H_{i}^{(j)}(\kappa u)}{j !}\left[\kappa h_{3}\left(u^{2}-1\right)+\kappa h_{4}\left(u^{3}-3 u\right)\right]^{j}
$$

where $H_{i}^{(j)}(\cdot)$ is the $j$ th-order derivative of $H_{i}(\cdot)$. As $n=1-4$, one has the first four-order Taylor series of $H_{i}(\tilde{x})$. Clearly, a higher order series provides a more accurate solution. For the $n$th order Taylor series of $H_{i}(\tilde{x})$, a set of simultaneous 
433

434

435

436

437

438

439

440

441

442

443

444

445

446

447

448

449

450

451

452

453

454

equations to solve the parameters of $\kappa, h_{3}$ and $h_{4}$ can be obtained if the expectation is applied to both sides of Eq. (33) with $i=2,3$ and 4, respectively. In this study, the derivation of the set of simultaneous equations for the 4th order Taylor series of $H_{i}(\tilde{x})$ is illustrated as an example $(n=4)$. Note that $E\left(u^{i}\right)=0$ for odd $i$ and $E\left(u^{i}\right)=(i-1) ! !$ for even $i$ are used to determine the moments of the standard Gaussian process $U(t)$ in the following derivation.

(1), $i=2$

In Eq. (33), $\quad H_{2}(\tilde{x})=\tilde{x}^{2}-1, \quad H_{2}(\kappa u)=\kappa^{2} u^{2}-1, \quad H_{2}^{(1)}(\kappa u)=2 \kappa u \quad$, and $H_{2}^{(2)}(\kappa u)=2$. Taking the expectation on both sides of Eq. (33) and making the simplification, one has

$$
\kappa^{2}\left(1+2 h_{3}^{2}+6 h_{4}^{2}\right)=1
$$

$$
\text { (2), } i=3
$$

In Eq. (33), $H_{3}(\tilde{x})=\tilde{x}^{3}-3 \tilde{x}, \quad H_{3}(\kappa u)=\kappa^{3} u^{3}-3 \kappa u, \quad H_{3}^{(1)}(\kappa u)=3 \kappa^{2} u^{2}-3$, $H_{3}^{(2)}(\kappa u)=6 \kappa u$, and $H_{3}^{(3)}(\kappa u)=6$. Taking the expectation on both sides of Eq. (33) and making the simplification using Eq. (34), one has

$$
\alpha_{3}=2 \kappa h_{3}\left(2+\kappa^{2}+18 \kappa^{2} h_{4}+42 \kappa^{2} h_{4}^{2}\right)
$$

$$
\text { (3), } i=4
$$

In Eq.

(33),

$$
H_{4}(\tilde{x})=\tilde{x}^{4}-6 \tilde{x}^{2}+3 \quad,
$$$$
H_{4}(\kappa u)=\kappa^{4} u^{4}-6 \kappa^{2} u^{2}+3,
$$

$H_{4}^{(1)}(\kappa u)=4 \kappa^{3} u^{3}-12 \kappa u \quad, \quad H_{4}^{(2)}(\kappa u)=12\left(\kappa^{2} u^{2}-1\right) \quad, \quad H_{4}^{(3)}(\kappa u)=24 \kappa u \quad, \quad$ and $H_{4}^{(4)}(\kappa u)=24$. Taking the expectation on both sides of Eq. (33) and making the simplification using Eq. (34), one has

$$
\begin{gathered}
\alpha_{4}=15-12 \kappa^{4}+\left(288-264 \kappa^{2}\right) \kappa^{2} h_{4} \\
+\left(936-864 \kappa^{2}\right) \kappa^{2} h_{4}^{2}-432 \kappa^{4} h_{4}^{3}-2808 \kappa^{4} h_{4}^{4}
\end{gathered}
$$


455

456

457

458

459

460

461

462

463

464

465

466

467

468

469

470

471

472
Eqs. (34), (35) and (36) can be used to obtain the parameters of $\kappa, h_{3}$ and $h_{4}$, which were introduced by Ditlevsen et al. (1996). The four sets of equations associated with the first four-order Taylor series of $H_{i}(\tilde{x})$ can be found in Yang and Tian (2015).

Alternatively, there is another way to infer nonlinear equations. The $i$ th moment on both sides of Eq. (17) is expressed by

$$
E\left(\tilde{x}^{i}\right)=E\left\{\kappa^{i}\left[u+h_{3}\left(u^{2}-1\right)+h_{4}\left(u^{3}-3 u\right)\right]^{i}\right\}
$$

The following equations are obtained by substituting $i=2,3$ and 4 into Eq. (37) respectively

$$
E\left(\tilde{x}^{2}\right)=\kappa^{2}\left(1+2 h_{3}^{2}+6 h_{4}^{2}\right)=1
$$

$$
\begin{gathered}
E\left(\tilde{x}^{4}\right)=\kappa^{4}\left(3+24 h_{4}+60 h_{3}^{2}+252 h_{4}^{2}+576 h_{3}^{2} h_{4}+\right. \\
\left.1296 h_{4}^{3}+60 h_{3}^{4}+2232 h_{3}^{2} h_{4}^{2}+3348 h_{4}^{4}\right)=\alpha_{4}
\end{gathered}
$$

Eqs. (38)-(40) can be found in Tognarelli et al. (1997) and Gurley (1997). Note that Eqs. (38) and (34) are same.

Substituting Eq. (38) into Eqs. (39) and (40), respectively, one has the following equations

$$
\begin{gathered}
\left.4 \kappa h_{3} \kappa^{2}\left(1+2 h_{3}^{2}+6 h_{4}^{2}\right)\right]+2 \kappa^{3} h_{3}+36 \kappa^{3} h_{3} h_{4}+84 \kappa^{3} h_{3} h_{4}^{2} \\
=2 \kappa h_{3}\left(2+\kappa^{2}+18 \kappa^{2} h_{4}+42 \kappa^{2} h_{4}^{2}\right)=\alpha_{3}
\end{gathered}
$$

$$
\begin{gathered}
\kappa^{4}\left[\beta+24 h_{4}+30\left(\frac{1}{\kappa^{2}}-1-6 h_{4}^{2}\right)+252 h_{4}^{2}+288 h_{4}\left(\frac{1}{\kappa^{2}}-1-6 h_{4}^{2}\right)\right. \\
\left.+1296 h_{4}^{3}+15\left(\frac{1}{\kappa^{2}}-1-6 h_{4}^{2}\right)^{2}+1116 h_{4}^{2}\left(\frac{1}{\kappa^{2}}-1-6 h_{4}^{2}\right)+3348 h_{4}^{4}\right] \\
=15-12 \kappa^{4}+\left(288-264 \kappa^{2}\right) \kappa^{2} h_{4}+\left(936-864 \kappa^{2}\right) \kappa^{2} h_{4}^{2} \\
-432 \kappa^{4} h_{4}^{3}-2808 \kappa^{4} h_{4}^{4}=\alpha_{4}
\end{gathered}
$$


473 Hence, Eqs. (41) and (42) show that the two sets of nonlinear equations are

474

475

476

477

478

479

480

481

482

483

484

485

486

487 Clearly, $\mu_{x}=\frac{1}{M} \sum_{m=1}^{M} \mu_{x}^{m}$ where $\mu_{x}$ is the mean for $X(t)$. The STD $\sigma_{x}^{m}$ varies equivalent.

\section{Appendix B}

Suppose wind pressure time history $X(t)$ and its standardized version $\tilde{X}(t)$ are composed of $M$ segments. If the $m$ th segment of $\tilde{X}(t), \tilde{X}^{m}(t)$, is used to estimate the peak value at $q$ fractile, $\tilde{x}_{p, q}^{m}$, through the peak CDF or Eq. (29), the averaged value is

$$
E\left[\tilde{x}_{p, q}\right]=\frac{1}{M} \sum_{m=1}^{M} \tilde{x}_{p, q}^{m}
$$

where $E[\cdot]$ denotes the averaging across the samples.

Accordingly, $x_{p, q}^{m}$, the peak value at $q$ fractile for the $m$ th segment of $X(t)$, $X^{m}(t)$, can be obtained from Eq. (30), i.e., $x_{p, q}^{m}=\mu_{x}^{m}+\tilde{x}_{p, q}^{m} \sigma_{x}^{m}$ where $\mu_{x}^{m}$ and $\sigma_{x}^{m}$ are the mean and STD of $X^{m}(t)$, respectively. The corresponding averaged value is

$$
E\left[x_{p, q}\right]=\frac{1}{M} \sum_{m=1}^{M}\left(\mu_{x}^{m}+\tilde{x}_{p, q}^{m} \sigma_{x}^{m}\right)
$$

488 slowly across the different segments. For example, the mean, maximum, minimum of

489 STD for tap 4 are $0.2,0.222$ and 0.174 , respectively. The corresponding coefficient of

490

491 variation is 0.04 . Hence, the following relation can be obtained for large $M$

$$
E\left[x_{p, q}\right] \approx \mu_{x}+E\left[\tilde{x}_{p, q}\right] \sigma_{x}
$$

492

where $\sigma_{x}$ is the STD for $X(t)$. 
Figure 1

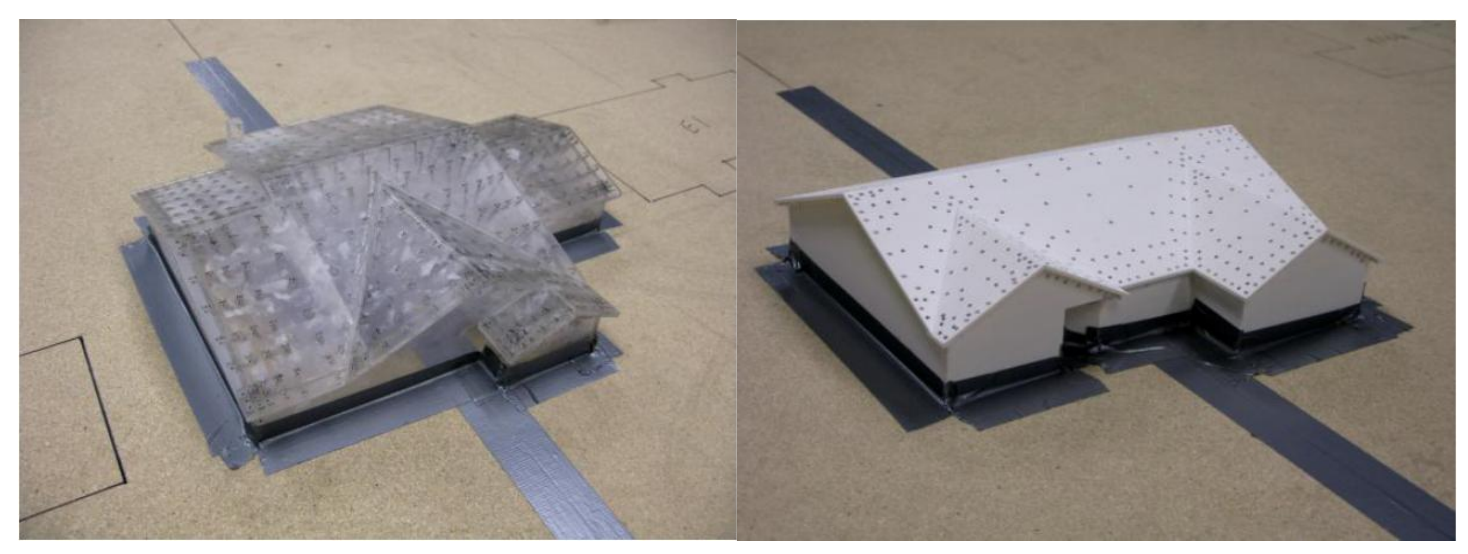

(a) FL27

(b) FL30

Figure 1 House models (Peng et al. 2014) 
Figure 2

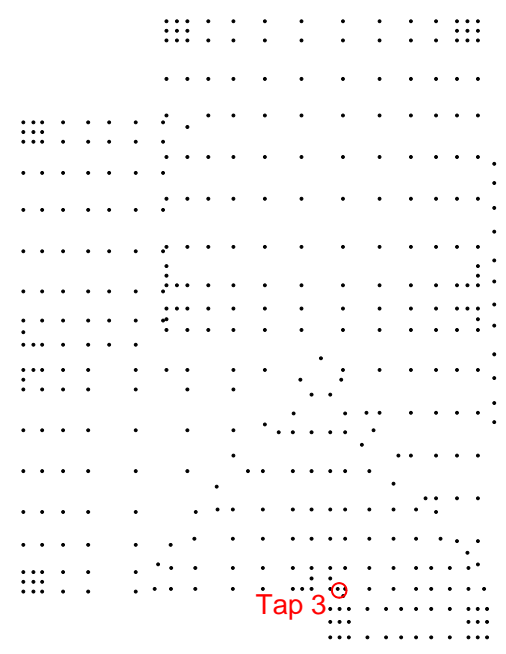

(a) FL27

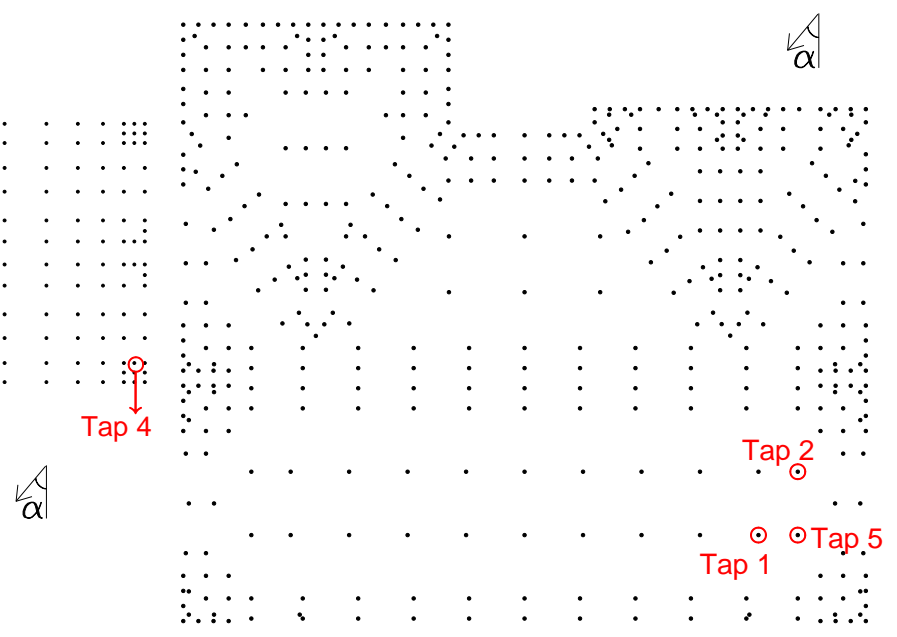

(b) FL30

Figure 2 Tap locations and wind direction for house models 
Figure 3

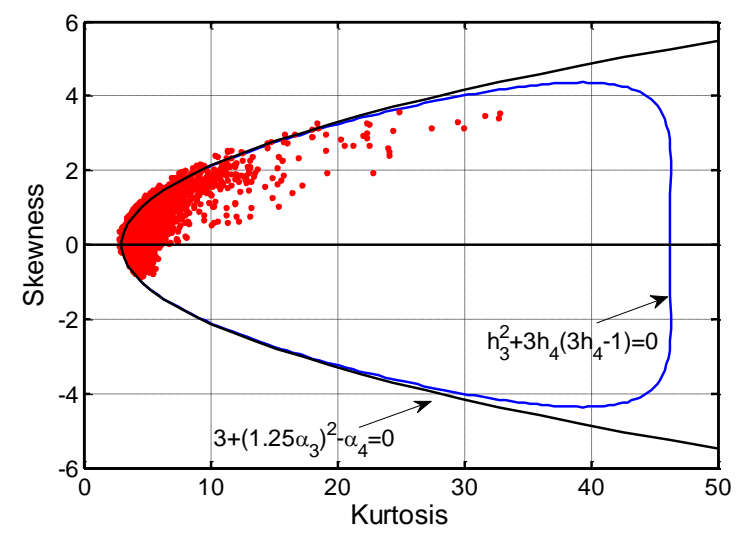

Figure 3 Skewness and kurtosis of wind pressure coefficients for all taps 


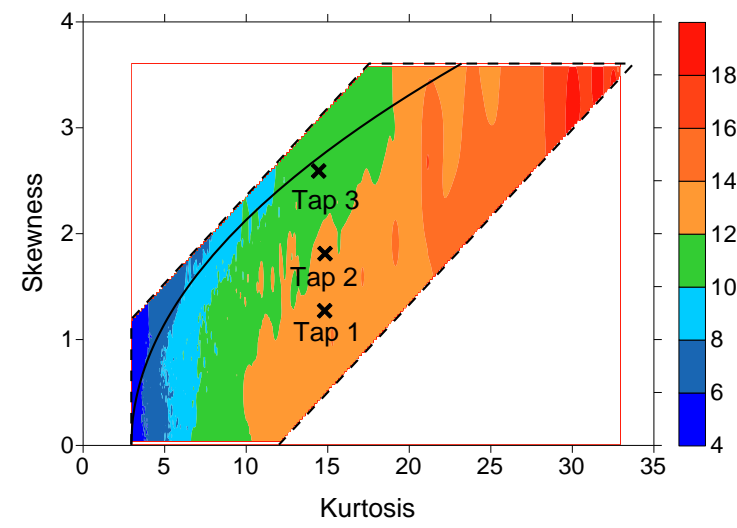

(a) Peak factor

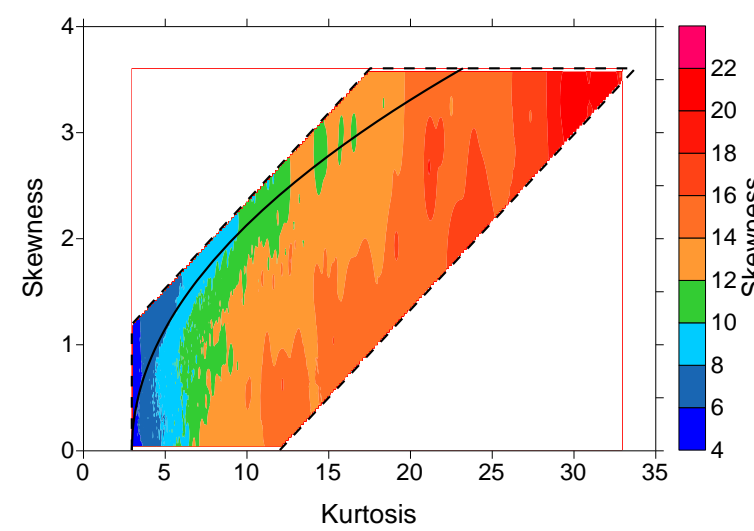

(c) $78 \%$ fractile

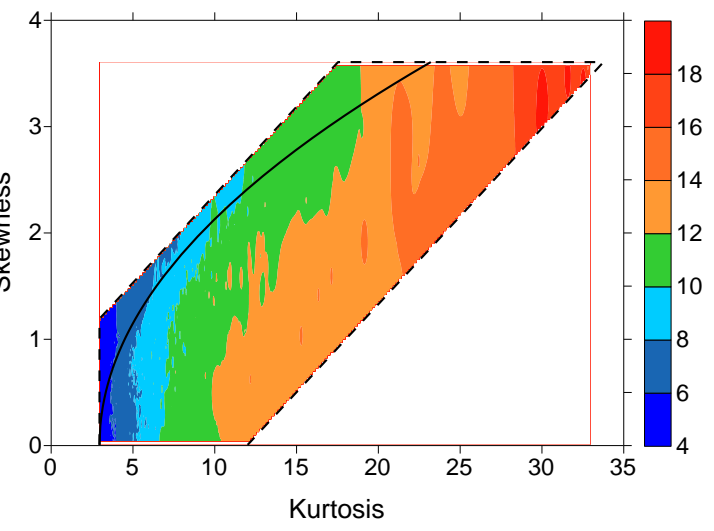

(b) $57 \%$ fractile

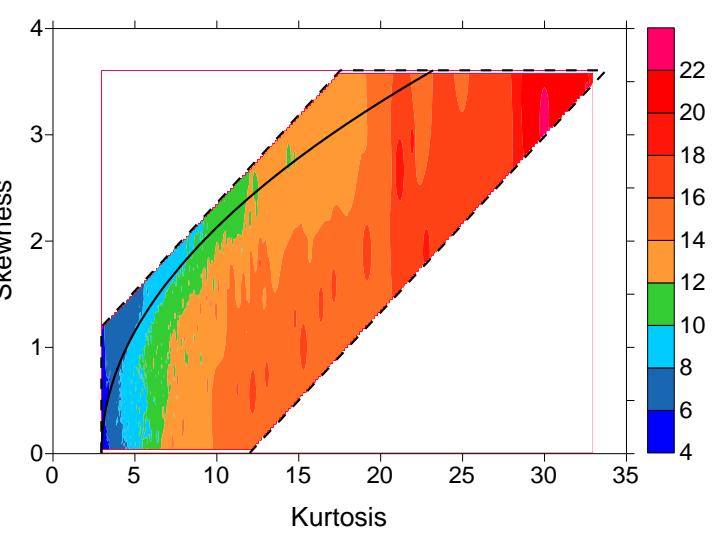

(d) $86 \%$ fractile

Figure 4 Peak factor and various fractile values based on wind tunnel data 
Figure 5

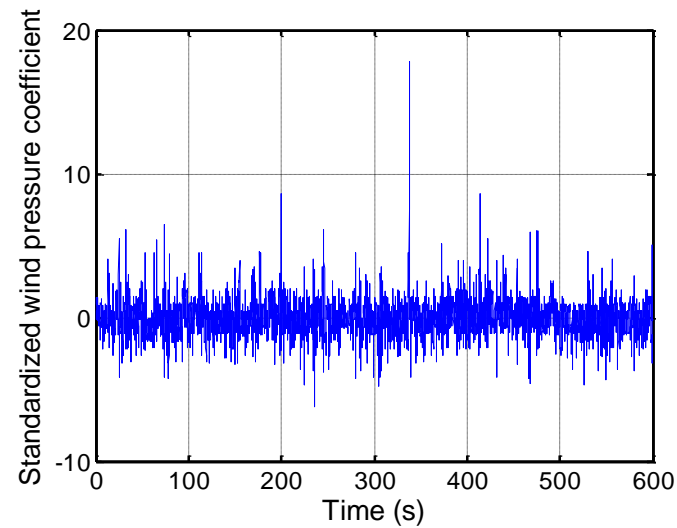

(a) tap 1

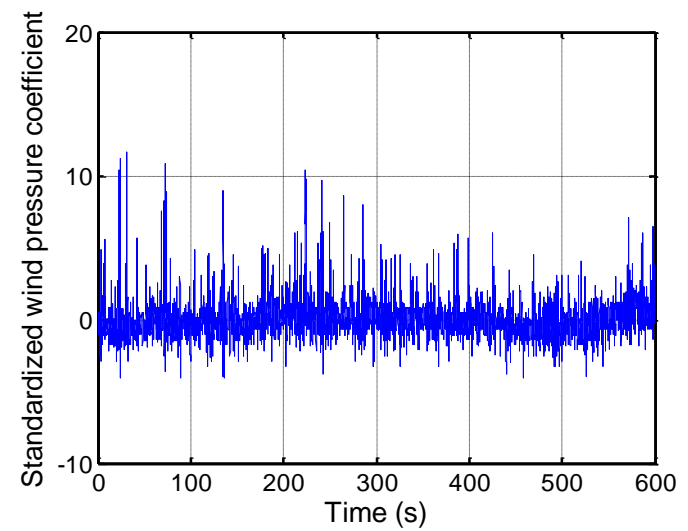

(b) tap 2

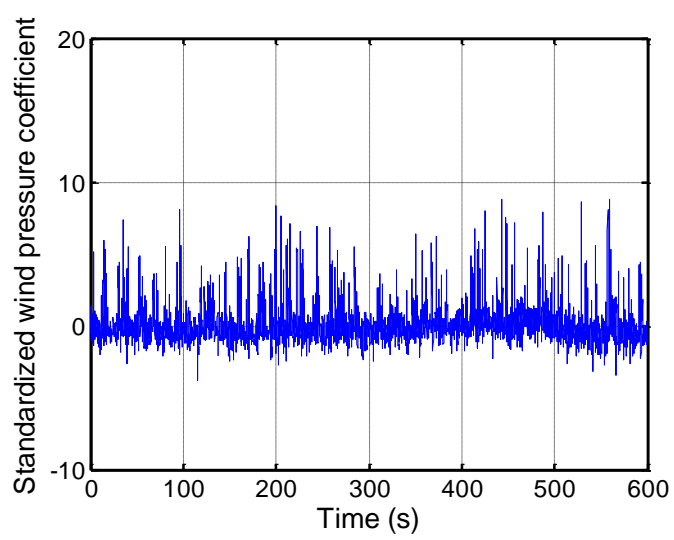

(c) $\operatorname{tap} 3$

Figure 5 Standardized time histories of wind pressure coefficients for taps 1-3 


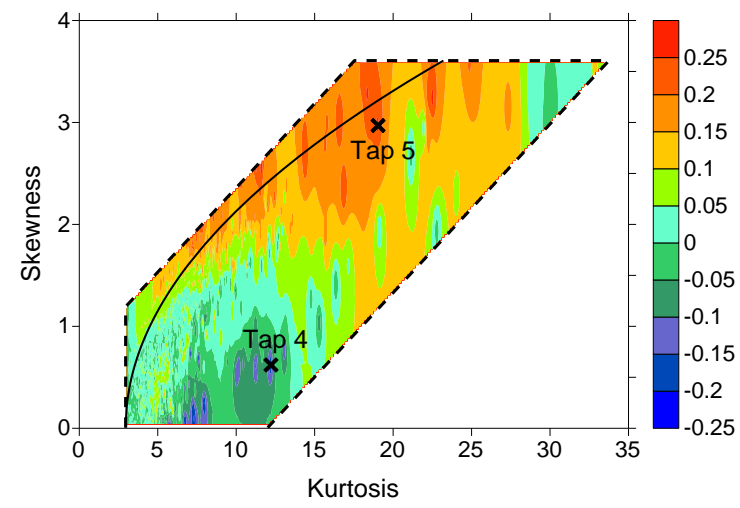

(a) Peak factor

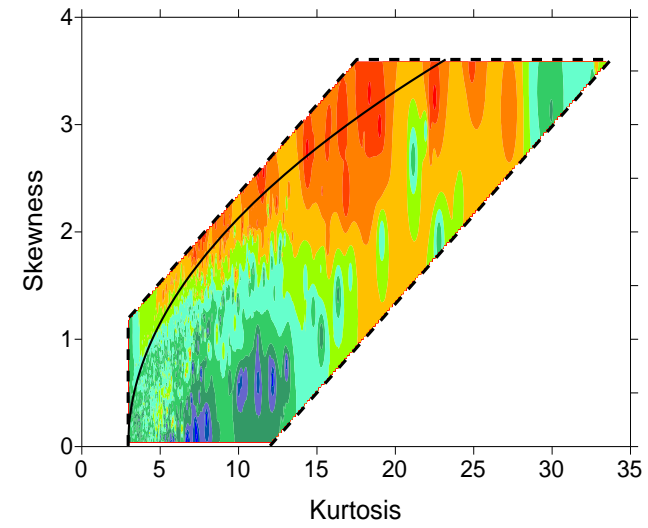

(b) $78 \%$ fractile
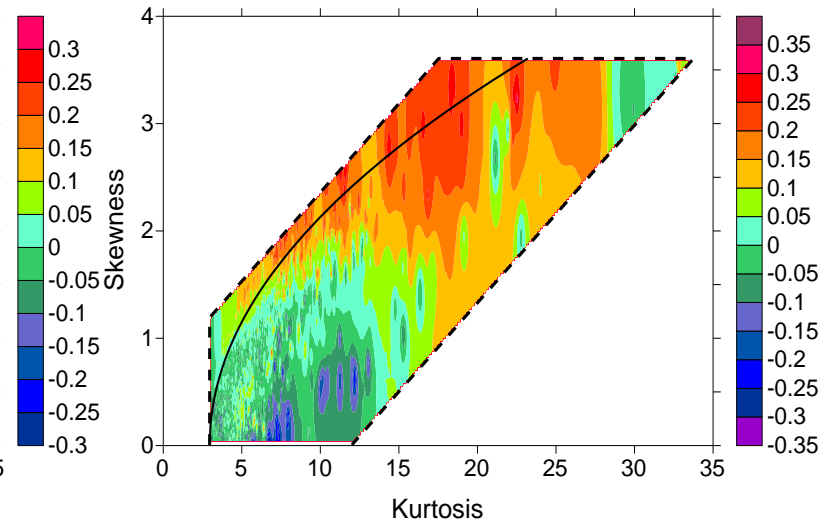

(c) $86 \%$ fractile

Figure 6 Contour of relative error of mean peak factors and various fractile values estimated by

HPM from 180 segments 
Figure 7

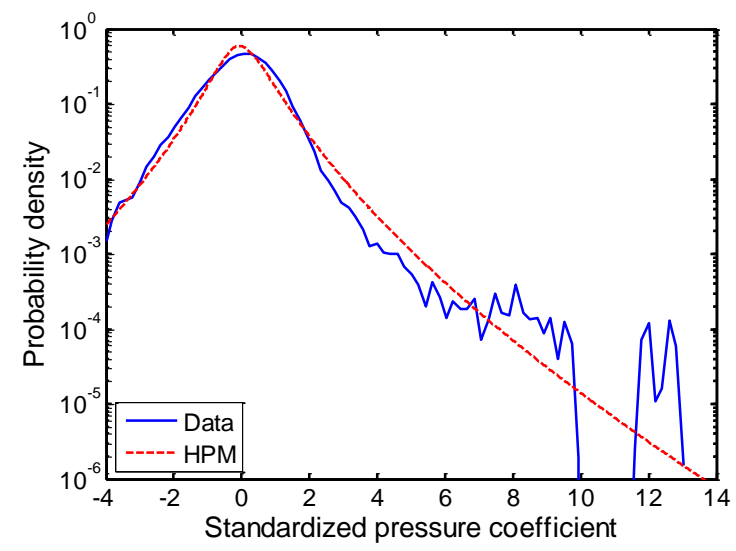

(a) Segment 1

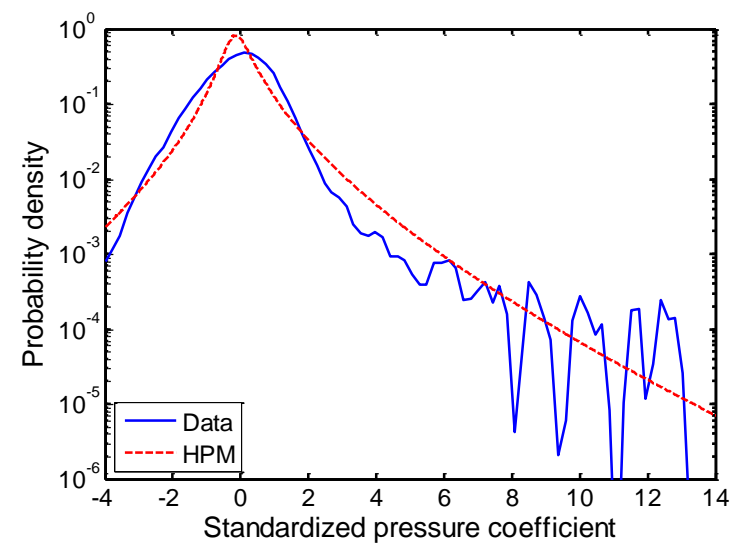

(b) Segment 2

Figure 7 Histogram and HPM PDFs of two 10-min segments for tap 4 
Figure 8

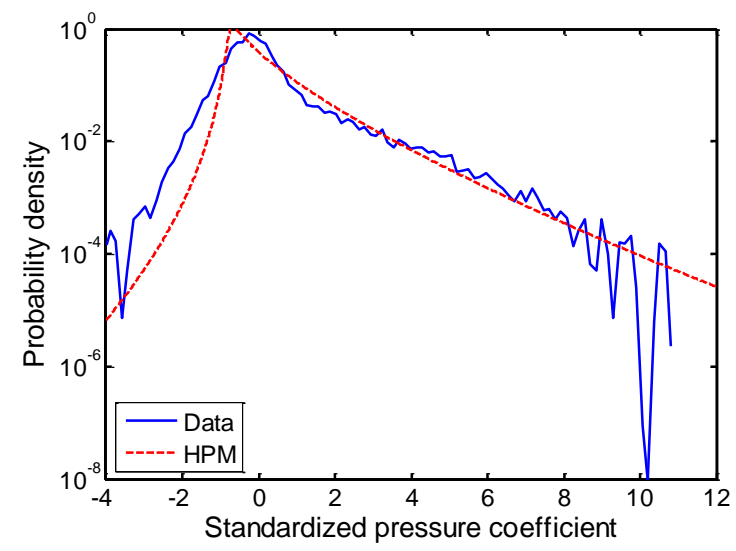

(a) Segment 1

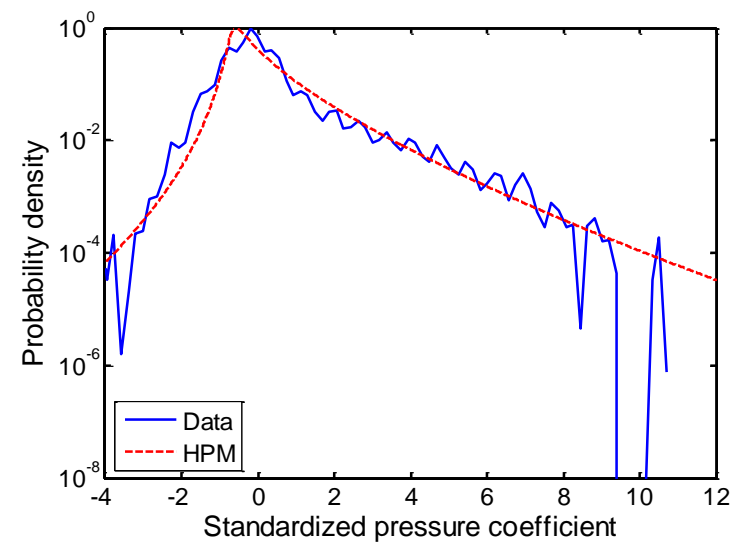

(b) Segment 2

Figure 8 Histogram and HPM PDFs of two 10-min segments for tap 5 
Figure 9

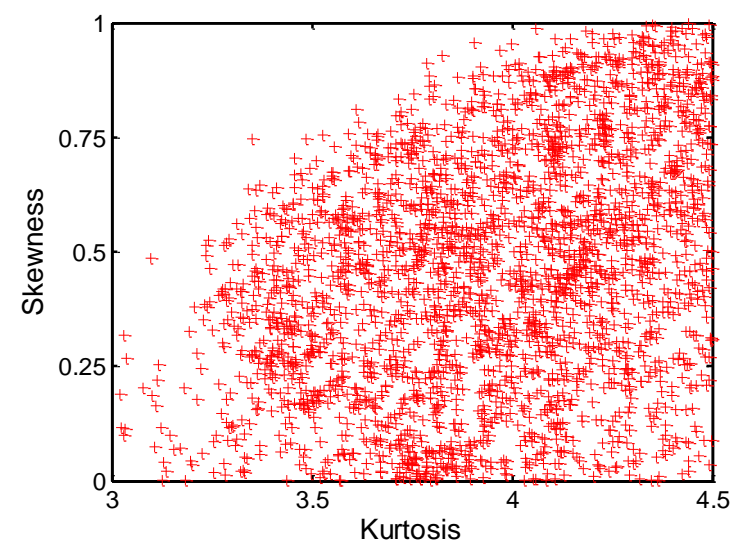

Figure 9 Scatter of skewness and kurtosis of wind pressure coefficients 


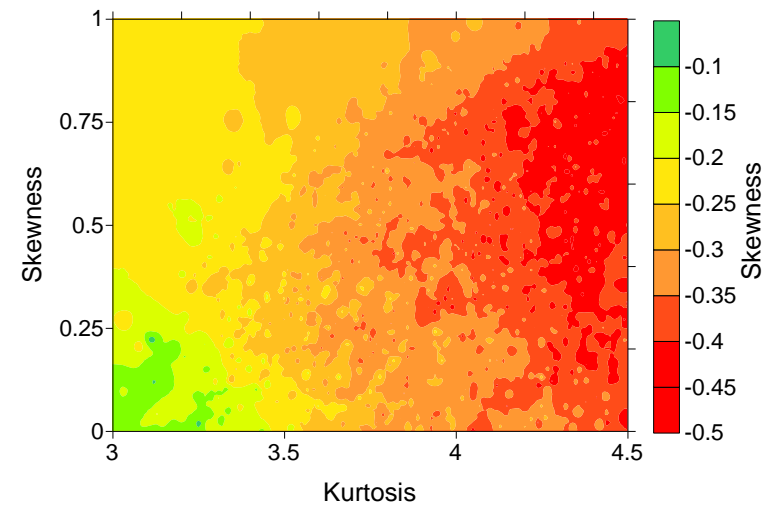

(a) Davenport's formula

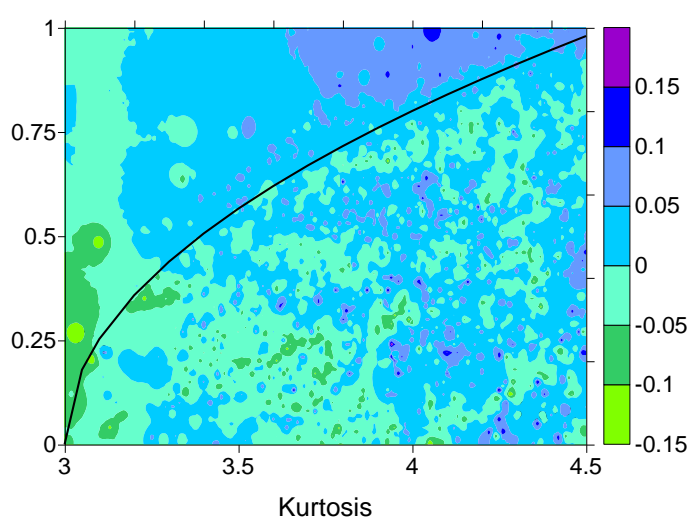

(b) HPM

Figure 10 Errors of peak factors by different formulas 


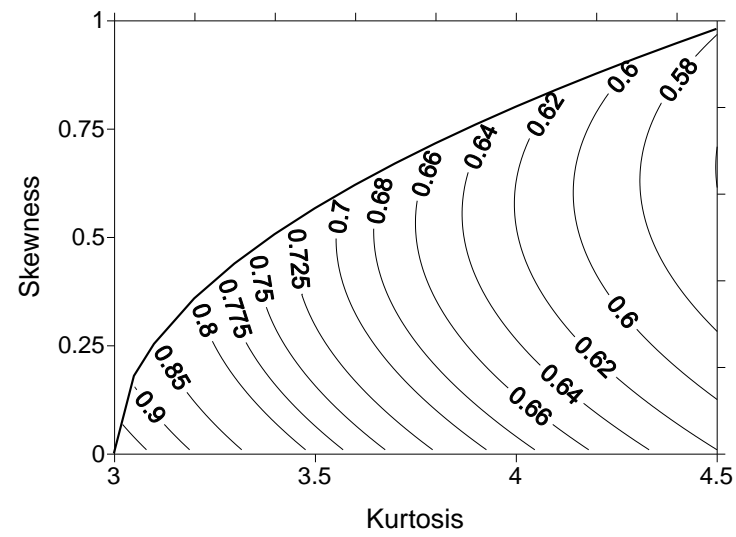

Figure 11 Ratios of peak factors calculated by Davenport's formula to those by HPM 
Table 1 Model, terrain and wind direction for the selected taps

\begin{tabular}{|c|c|c|c|}
\hline Tap & Model & Terrain & Wind direction \\
\hline 1 & FL30 & I & $120^{\circ}$ \\
\hline 2 & FL30 & H & $130^{\circ}$ \\
\hline 3 & FL27 & I & $125^{\circ}$ \\
\hline 4 & FL27 & I & $130^{\circ}$ \\
\hline 5 & FL30 & I & $120^{\circ}$ \\
\hline
\end{tabular}


Table 2

Table 2 Higher moments and peak factors for taps 1-3

\begin{tabular}{|c|c|c|c|}
\hline Tap & Skewness & Kurtosis & Peak factor \\
\hline 1 & 1.272 & 14.810 & 13.738 \\
\hline 2 & 1.812 & 14.832 & 12.799 \\
\hline 3 & 2.592 & 14.445 & 10.577 \\
\hline
\end{tabular}


Table 3 Percentages of taps associated with different relative error ranges for peak factor and various fractile values

\begin{tabular}{|c|c|c|c|c|c|c|}
\hline \multirow{2}{*}{} & \multicolumn{7}{|c|}{ Relative error ranges } \\
\cline { 2 - 7 } & $<-20 \%$ & $-20 \% \sim-10 \%$ & $-10 \% \sim 0 \%$ & $0 \% \sim 10 \%$ & $10 \% \sim 20 \%$ & $>20 \%$ \\
\hline Peak factor & $0.26 \%$ & $5.02 \%$ & $30.64 \%$ & $48.43 \%$ & $14.46 \%$ & $1.19 \%$ \\
\hline $78 \%$ fractile & $1.22 \%$ & $11.50 \%$ & $33.16 \%$ & $40.12 \%$ & $12.50 \%$ & $1.50 \%$ \\
\hline $86 \%$ fractile & $2.59 \%$ & $14.67 \%$ & $31.73 \%$ & $36.99 \%$ & $11.93 \%$ & $2.10 \%$ \\
\hline
\end{tabular}

